\title{
Telemedicine Tools for Patients and Providers: Systematic Review
}

\author{
Jacqueline Sly ${ }^{1}$, BSN, RN; Beth Clarke ${ }^{1}$, BSN, RN; Andreina Sanabria ${ }^{2}$, BSN, RN \\ ${ }^{1}$ Connell School of Nursing, Boston College, Chesnut Hill, MA, United States \\ ${ }^{2}$ Woods College of Advancing Studies, Boston College, Chestnut Hill, MA, United States
}

\section{Corresponding Author:}

Andreina Sanabria, BSN, RN

Woods College of Advancing Studies

Boston College

140 Commonwealth Avenue

Chestnut Hill, MA, 02467

United States

Phone: 9559117

Email: $\underline{\text { sanabran@bc.edu }}$

\section{Abstract}

Background: From the 1991 Institute of Medicine (IOM) Landmark Report, The Computer Based Patient Record: An Essential Technology for Health Care (Dick, Steen, \& Detmer, 1997) which called for computer based patient records, to the 2009 Health Information Technology for Economic and Clinical Health (HITECH) Act, health care has been integrating technology into our delivery systems and transforming the landscape of health care delivery (Wager, Lee, \& Glaser, 2017). Telemedicine (also called telemonitoring, telehealth, remote monitoring, eHealth) has brought about new methods of care delivery. These methods will hopefully demonstrate cost effective outcomes as well as new levels of quality. Under the influence of The Centers for Medicare and Medicaid Services, The 2015 Medicare Access and CHIP Reauthorization Act (MACRA) was enacted which pushes the health care industry to improve quality and value. By the end of 2018 , it is expected that $50 \%$ of alternative payment models will have quality and value tied to them and $90 \%$ of fee-for-service will do the same. It is feasible that telehealth could be the link to new health care delivery and payment models. This paper evaluates some of the most recent literature regarding telemedicine to elucidate quality within the domains of cost-effectiveness, hospitalization, mortality, patient adherence, and patient satisfaction.

Objective: To evaluate the effectiveness of telemedicine strategies with respect to cost-effectiveness of care, hospitalizations, mortality, patient adherence, and satisfaction.

Methods: The Google Scholar search engine was used to find articles published between 2015 and 2018 so that preliminary observations could be made regarding the telemedicine literature. Once this was done, a logical search process was used to locate articles using two research databases: Cumulative Index to Nursing and Allied Health Literature (CINAHL) and PubMed (MEDLINE). The titles and abstracts of the records identified were independently screened by four investigators. Five reviewers screened 58 abstracts to determine relevance and significance relative to the research objectives. The final group analysis was 29 articles. Main outcome measures included economic, clinical, and satisfaction outcomes.

Results: Our results showed that examining telemedicine interventions that were either patient or provider driven appeared to improve outcomes related to patient adherence, patient satisfaction, cost-effectiveness, and had a favorable effect in terms of decreasing hospitalizations. Only two articles that were reviewed addressed mortality, so the investigators could not describe results on its effect. Although there are barriers to adherence there is much progress in the field of telemedicine that aids in outcomes, both positive and negative.

Conclusions: The literature is clear that telemedicine is linked to improved outcomes when applied with a sound strategic plan. Patients who adhere to telemedicine interventions appear to show significantly better satisfaction, quality of life, reduced hospitalizations, and related health care costs. When providers take greater responsibility and initiative with telemedicine interventions, this contributes to those patient outcomes. Our review of the literature suggests that the quality of the implementation strategy was linked with better outcomes. Telemedicine appears to hold promise as a cost effective, convenient tool for both patients and providers.

(iproc 2018;4(2):e11866) doi: $\underline{10.2196 / 11866}$

\section{KEYWORDS}

telemedicine; chronic disease; evidenced-based; reimbursement 
Edited by T Hale; this is a non-peer-reviewed article. Submitted 07.08.18; accepted 29.08.18; published 17.09.18.

Please cite as:

Sly J, Clarke B, Sanabria A

Telemedicine Tools for Patients and Providers: Systematic Review iproc 2018;4(2):e11866

URL: http://www.iproc.org/2018/2/e11866/

doi: $\underline{10.2196 / 11866}$

PMID:

(CJacqueline Sly, Beth Clarke, Andreina Sanabria. Originally published in Iproceedings (http://www.iproc.org), 17.09.2018. This is an open-access article distributed under the terms of the Creative Commons Attribution License (https://creativecommons.org/licenses/by/4.0/), which permits unrestricted use, distribution, and reproduction in any medium, provided the original work, first published in Iproceedings, is properly cited. The complete bibliographic information, a link to the original publication on http://www.iproc.org/, as well as this copyright and license information must be included. 